\title{
A viability result for functional differential inclusions in Banach spaces
}

\author{
Myelkebir Aitalioubrahim
}




\title{
A VIABILITY RESULT FOR FUNCTIONAL DIFFERENTIAL INCLUSIONS IN BANACH SPACES
}

\author{
MYELKEBIR AITALIOUBRAHIM \\ Received 16 February, 2011
}

\begin{abstract}
This paper is concerned with the existence of viable or monotone solutions of a first order functional differential inclusion in a separable Banach space. We consider two cases with moving constraints.
\end{abstract}

2000 Mathematics Subject Classification: 34A60; 49J52

Keywords: multifunction, measurability, selection, functional differential inclusion

\section{INTRODUCTION}

Let $E$ be a separable Banach space with norm $\|$.$\| . For a segment I$ in $\mathbb{R}$, we denote by $\mathcal{C}(I, E)$ the Banach space of continuous functions from $I$ to $E$ equipped with the norm $\|x(.)\|_{\infty}:=\sup \{\|x(t)\| ; t \in I\}$. For a positive number $a$, we put

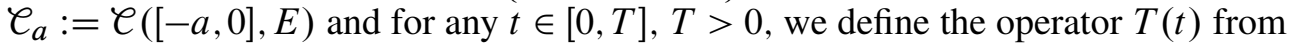
$\mathcal{C}([-a, T], E)$ to $\mathcal{C}_{a}$ with $(T(t)(x())).(s):=(T(t) x)(s):=x(t+s), s \in[-a, 0]$. For a given nonempty subset $K$ of $E$ we introduce the set $\mathcal{K}_{0}:=\left\{\varphi(.) \in \mathcal{C}_{a} ; \varphi(0) \in K\right\}$.

In this paper, we shall prove local existence of the solutions to the following functional differential inclusions:

$$
\begin{gathered}
\left\{\begin{array}{l}
\dot{x}(t) \in F(t, T(t) x), \quad \text { a.e. on }[0, T] ; \\
x(s)=\varphi(s), \quad \forall s \in[-a, 0] ; \\
x(t) \in C(t), \quad \forall t \in[0, T] ;
\end{array}\right. \\
\left\{\begin{array}{l}
\dot{x}(t) \in F(t, T(t) x), \quad \text { a.e. on }[0, T] ; \\
x(s)=\varphi(s), \quad \forall s \in[-a, 0] ; \\
x(t) \in P(x(s)), \quad \forall s \in[0, T], \quad \forall t \in[s, T] ;
\end{array}\right.
\end{gathered}
$$

where $F$ is a closed multifunction, measurable with respect to the first argument and Lipschitz continuous with respect to the second argument, $C$ and $P$ are two set-valued maps, and $\varphi$ is a given function in $\ell_{a}$.

This kind of problems was initiated by Haddad [5, 6], in the case when $F$ is globally upper semi-continuous and takes convex compact values and $C$ is fixed. Then 
this line of research was pursued by several authors. Regarding the existence of solutions in a separable Banach space, we refer to Syam [9], where two results are given: the first one deals with a fixed convex constraint $C$ and a globally measurable multifunction $F$ which is upper semi-continuous in its second argument, while in the second one the constraint is convex and moving, but $F$ is globally upper semicontinuous. See also the work of Gavioli and Malaguti [4].

Duc Ha, in [3], established the existence of viable solutions of (1.1) without memory in a Banach space when $C$ is fixed and $F$ is closed, integrably bounded, measurable with respect to the first argument and Lipschitz continuous with respect to the second argument. The author established a multi-valued version of Larrieu's work [7], assuming the following tangential condition:

$$
\liminf _{h \mapsto 0^{+}} \frac{1}{h} e\left(x+\int_{t}^{t+h} F(s, x) d s, C\right)=0,
$$

where $e(.,$.$) denotes the Hausdorff excess. Duc Ha's result has been extended by$ Lupulescu and Necula [8] to functional differential inclusions, but under the same hypotheses on $F$, assuming that $C$ is always fixed. They used a suitable adapted form of the tangential condition of (1.3).

Our work extends the results of $[3,8]$. Indeed, we get existence results, in a separable Banach space, for functional differential inclusions, with a not necessarily convex constraint which depends on time or on the variable $x \in E$. The right-hand side is not necessarily convex. As is known, viability problems need tangential conditions. For problems (1.1) and (1.2), we shall use a tangency condition which is weaker than the one used in $[3,8]$.

The paper is organized as follows. In Section 2, we recall some preliminary facts that we need in the sequel. In Section 3, we give some auxiliary results. In Section 4, we prove the existence of solutions for (1.1), while in Section 4 we establish the existence of monotone solutions for (1.2).

\section{Statement of the MAin Result}

For the purpose of measurability, $E$ (resp. $\Omega \subset E$ ) is endowed with the $\sigma$ algebra $B(E)$ (resp. $B(\Omega)$ ) of Borel subsets for the strong topology and $[0,1]$ is endowed with Lebesgue measure and the $\sigma$-algebra of Lebesgue measurable subsets. For $x \in E$ and $r>0$, let $B(x, r):=\{y \in E ;\|y-x\|<r\}$ be the open ball centered at $x$ with radius $r$ and let $\bar{B}(x, r)$ be its closure and put $B=B(0,1)$. For $\varphi(.) \in \mathcal{C}_{a}$ let $B_{a}(\varphi(), r):.=\left\{\psi(.) \in \mathcal{C}_{a} ;\|\varphi(.)-\psi(.)\|_{\infty}<r\right\}$ and let $\bar{B}_{a}(\varphi(), r$. be its closure. For $x \in E$ and for nonempty subsets $A, B$ of $E$, we denote by $d_{A}(x)$ or $d(x, A)$ the real inf $\{\|y-x\| ; y \in A\}, e(A, B):=\sup \left\{d_{B}(x) ; x \in A\right\}$ and $H(A, B)=\max \{e(A, B), e(B, A)\}$. For a multifunction $F$ we denote by $\operatorname{Gr}(F)$ its graph. A multifunction is said to be measurable if its graph is measurable. For more 
detail on measurability theory, we refer the reader to the book of Castaing-Valadier [2].

Let us recall the following Lemmas that will be used in the sequel. For the proofs, we refer the reader to [10].

Lemma 1. Let $\Omega$ be a nonempty set in $E$. Assume that $F:[a, b] \times \Omega \rightarrow 2^{E}$ is a multifunction with nonempty closed values satisfying:

- For every $x \in \Omega, F(., x)$ is measurable on $[a, b]$;

- For every $t \in[a, b], F(t,$.$) is (Hausdorff) continuous on \Omega$.

Then for any measurable function $x():.[a, b] \rightarrow \Omega$, the multifunction $F(., x()$.$) is$ measurable on $[a, b]$.

Lemma 2. Let $G:[a, b] \rightarrow 2^{E}$ be a measurable multifunction and $y():.[a, b] \rightarrow$ $E$ a measurable function. Then for any positive measurable function $r():.[a, b] \rightarrow$ $\mathbb{R}^{+}$, there exists a measurable selection $g($.$) of G$ such that for almost all $t \in[a, b]$

$$
\|g(t)-y(t)\| \leq d(y(t), G(t))+r(t) .
$$

Let us introduce the following hypotheses which we shall use throughout this paper.

\section{Hypothesis (H).}

(H1) $C:[-1,1] \rightarrow 2^{E}$ is a lower semicontinuous set-valued map with compact graph and $\mathcal{K}:[-1,1] \rightarrow \mathcal{C}_{a}$ is a set-valued map defined by $\mathcal{K}(t)=\{\varphi \in$ $\left.\bigodot_{a}, \varphi(0) \in C(t)\right\}$

(H2) $F: \operatorname{Gr}(\mathcal{K}) \rightarrow 2^{E}$ is a set-valued map with nonempty closed values satisfying

(i) $t \mapsto F(t, \psi)$ is measurable,

(ii) There exists a function $m(.) \in L^{1}\left([0,1], \mathbb{R}^{+}\right)$such that for all $t \in[0,1]$ and $\psi_{1}, \psi_{2} \in \mathcal{K}(t)$

$$
H\left(F\left(t, \psi_{1}\right), F\left(t, \psi_{2}\right)\right) \leq m(t)\left\|\psi_{1}-\psi_{2}\right\|_{\infty},
$$

(iii) There exists $g(.) \in L^{1}\left([0,1], \mathbb{R}^{+}\right)$such that for all $t \in[0,1]$ and $\psi \in$ $\mathcal{K}(t)$

$$
\|F(t, \psi)\|:=\sup _{y \in F(t, \psi)}\|y\| \leq g(t) .
$$

Hypothesis (A).

(A1) $K$ is a nonempty compact subset in $E$ and $P: E \rightarrow 2^{K}$ is a lower semicontinuous set-valued map satisfying

(i) For all $x \in K, x \in P(x)$,

(ii) For all $x \in K$ and all $y \in P(x)$ we have $P(y) \subseteq P(x)$; 
(A2) $F:[-1,1] \times \mathcal{K}_{0} \rightarrow 2^{E}$ is a set-valued map with nonempty closed values satisfying

(i) For each $\psi \in \mathcal{K}_{0}, t \mapsto F(t, \psi)$ is measurable,

(ii) There exists a function $m(.) \in L^{1}\left([0,1], \mathbb{R}^{+}\right)$such that for all $t \in[0,1]$ and for all $\psi_{1}, \psi_{2} \in \mathcal{K}_{0}$

$$
H\left(F\left(t, \psi_{1}\right), F\left(t, \psi_{2}\right)\right) \leq m(t)\left\|\psi_{1}-\psi_{2}\right\|_{\infty},
$$

(iii) There exists $g(.) \in L^{1}\left([0,1], \mathbb{R}^{+}\right)$such that for all $t \in[0,1]$ and for all $\psi \in \mathcal{K}_{0}$

$$
\|F(t, \psi)\| \leq g(t) .
$$

In the rest of the paper, fix $\varphi \in \mathcal{C}_{a}$ such that $\varphi(0) \in C(0)$ for Problem (1.1) and $\varphi(0) \in K$ for Problem (1.2). For $\varepsilon>0$ set,

$$
\begin{gathered}
\eta(\varepsilon):=\sup \{\rho \in] 0, \varepsilon]:\left|\int_{t_{1}}^{t_{2}} g(\tau) d \tau\right|<\varepsilon,\left\|\varphi\left(t_{1}\right)-\varphi\left(t_{2}\right)\right\|<\varepsilon \\
\left.\quad \text { and }\left|\int_{t_{1}}^{t_{2}} m(\tau) d \tau\right|<\varepsilon \text { if }\left|t_{1}-t_{2}\right| \leq \rho\right\} .
\end{gathered}
$$

In the sequel we need the following notation. For given measurable functions $y():.[0,1] \rightarrow E$ and $r():.[0,1] \rightarrow \mathbb{R}^{+}$set

$$
\begin{gathered}
S_{y, r}(\psi)=\left\{f \in L^{1}([-1,1], E): f(s) \in F(s, \psi) \text { for all } s \in[-1,1]\right. \text { and } \\
\|f(s)-y(s)\| \leq d(y(s), F(s, \psi))+r(s) \text { for all } s \in[0,1]\}
\end{gathered}
$$

where $\psi \in \mathscr{C}_{a}$. Remark that the set $S_{y, r}(\psi)$ is nonempty if we take into account that $F$ satisfies the condition (H2) or (A2). Indeed, this fact can be trivially deduced from Lemma 1 and Lemma 2.

We are now ready to introduce the tangential conditions used in this work.

(H3) (Tangential condition) For all measurable functions $y():.[0,1] \rightarrow E, r($.$) :$ $[0,1] \rightarrow \mathbb{R}^{+}$and for every $t \in[0,1]$ and $\psi \in \mathcal{K}(t)$, there exists $f \in S_{y, r}(\psi)$ such that

$$
\liminf _{h \mapsto 0^{+}} \frac{1}{h} d\left(\psi(0)+\int_{t}^{t+h} f(s) d s, C(t+h)\right)=0 .
$$

(A3) (Tangential condition) For all measurable functions $y():.[0,1] \rightarrow E, r($.$) :$ $[0,1] \rightarrow \mathbb{R}^{+}$and for every $(t, \psi) \in[0,1] \times \mathcal{K}_{0}$, there exists $f \in S_{y, r}(\psi)$ such that

$$
\liminf _{h \mapsto 0^{+}} \frac{1}{h} d\left(\psi(0)+\int_{t}^{t+h} f(s) d s, P(\psi(0))\right)=0 .
$$


These tangential conditions are weaker than the ones used in $[3,8]$. It is important to notice here that, if $F$ and $C$ do not depend on time, (H3) and (A3) are equivalent to

$$
F(\psi) \cap T_{C}(\psi(0)) \neq \varnothing \text { and } F(\psi) \cap T_{P(\psi(0))}(\psi(0)) \neq \varnothing
$$

respectively, where $T_{K}(x)$ is the contingent cone at $x$ to $K$ and $\varphi(.) \in \mathcal{C}_{a}$. However, in this case the condition (1.3) is equivalent to $F(x) \subseteq T_{C}(x)$.

In the next sections, we shall prove the following theorems.

Theorem 1. If assumptions (H1)-(H3) are satisfied, then for all $\left(z_{0}, \varphi\right) \in E \times$ $\ell_{a}$ such that $\varphi(0)=z_{0} \in C(0)$, there exist $T>0$ and a continuous function $x($.$) :$ $[-a, T] \rightarrow E$ which is absolutety continuous on $[0, T]$ and it is a solution of (1.1).

Theorem 2. If assumptions (A1)-(A3) are satisfied, then for all $\left(z_{0}, \varphi\right) \in K \times \mathcal{K}_{0}$ such that $\varphi(0)=z_{0}$, there exist $T>0$ and a continuous function $x():.[-a, T] \rightarrow E$ which is absolutely continuous on $[0, T]$ and it is a monotone solution of (1.2).

\section{Preliminary Results}

In this section, we shall prove some auxiliary results that will be used in the next sections. Consider first the following hypotheses which we shall use throughout this section.

Hypothesis (C).

(C1) $C:[-1,1] \rightarrow 2^{E}$ is a lower semicontinuous set-valued map with compact graph;

(C2) $F: G r(C) \rightarrow 2^{E}$ is a set-valued map with nonempty closed values satisfying

(i) $t \mapsto F(t, x)$ is measurable,

(ii) There exists a function $m(.) \in L^{1}\left([0,1], \mathbb{R}^{+}\right)$such that for all $t \in[0,1]$ and $x_{1}, x_{2} \in \mathcal{C}(t)$

$$
H\left(F\left(t, x_{1}\right), F\left(t, x_{2}\right)\right) \leq m(t)\left\|x_{1}-x_{2}\right\|,
$$

(iii) There exists $g(.) \in L^{1}\left([0,1], \mathbb{R}^{+}\right)$such that for all $t \in[0,1]$ and $x \in \mathcal{C}(t)$

$$
\|F(t, x)\| \leq g(t)
$$

(C3) (Tangential condition) For all measurable functions $y():.[0,1] \rightarrow E, r($.$) :$ $[0,1] \rightarrow \mathbb{R}^{+}$and for every $t \in[0,1]$ and $x \in C(t)$, there exists $f \in S_{y, r}(x)$ such that

$$
\liminf _{h \mapsto 0^{+}} \frac{1}{h} d\left(x+\int_{t}^{t+h} f(s) d s, C(t+h)\right)=0 .
$$


Hypothesis (L).

(L1) $K$ is a nonempty compact subset in $E$ and $P: E \rightarrow 2^{K}$ is a lower semicontinuous set-valued map satisfying

(i) For all $x \in K, x \in P(x)$,

(ii) For all $x \in K$ and all $y \in P(x)$ we have $P(y) \subseteq P(x)$;

(L2) $F:[-1,1] \times K \rightarrow 2^{E}$ is a set-valued map with nonempty closed values satisfying

(i) For each $x \in K, t \mapsto F(t, x)$ is measurable,

(ii) There exists a function $m(.) \in L^{1}\left([0,1], \mathbb{R}^{+}\right)$such that for all $t \in[0,1]$ and for all $x_{1}, x_{2} \in K$

$$
H\left(F\left(t, x_{1}\right), F\left(t, x_{2}\right)\right) \leq m(t)\left\|x_{1}-x_{2}\right\|,
$$

(iii) There exists $g(.) \in L^{1}\left([0,1], \mathbb{R}^{+}\right)$such that for all $t \in[0,1]$ and for all $x \in K$

$$
\|F(t, x)\| \leq g(t)
$$

(L3) (Tangential condition) For all measurable functions $y():.[0,1] \rightarrow E, r($.$) :$ $[0,1] \rightarrow \mathbb{R}^{+}$and for every $(t, x) \in[0,1] \times K$, there exists $f \in S_{y, r}(x)$ such that

$$
\liminf _{h \mapsto 0^{+}} \frac{1}{h} d\left(x+\int_{t}^{t+h} f(s) d s, P(x)\right)=0 .
$$

In the following, let $b \in] 0,1\left[\right.$ be fixed and, for all $t_{0} \in[0, b]$ set $\left[t_{0}, b_{0}\right]=\left[t_{0}, t_{0}+\right.$ $\left.\frac{1}{4} \eta\left(\frac{\varepsilon}{4}\right)\right] \cap[0, b]$.

Lemma 3. If assumptions (C1)-(C3) are satisfied, then for all $0<\varepsilon<a, t_{0} \in[0, b]$ and for all measurable function $y():.[0, b] \rightarrow E$, there exists $\eta>0(\eta<\varepsilon)$ such that for all $t \in\left[t_{0}, b_{0}\right]$ and $x \in C(t)$, there exist $f \in S_{y,(2 m(.)+1) \varepsilon}(x), h_{t, x} \in\left[\eta, \frac{1}{4} \eta\left(\frac{\varepsilon}{4}\right)\right]$ and $u \in \varepsilon B$ such that

$$
\left(x+h_{t, x} u+\int_{t}^{t+h_{t, x}} f(s) d s\right) \in C\left(t+h_{t, x}\right) .
$$

Proof. Let $0<\varepsilon<a, t_{0} \in[0, b]$ and $y():.[0, b] \rightarrow E$ be a measurable function. Consider $(s, z) \in M:=\{(\bar{s}, \bar{z}) / \bar{s} \in[0, b]$ and $\bar{z} \in C(\bar{s})\}$. Note that if the subset $M$ of $\operatorname{Gr}(C)$ is closed then it is compact. By the tangential condition, there exists $f_{s, z} \in$ $S_{y, \frac{\varepsilon}{4}}(z)$ such that

$$
\liminf _{h \mapsto 0^{+}} \frac{1}{h} d\left(z+\int_{s}^{s+h} f_{s, z}(\tau) d \tau, C(s+h)\right)=0 .
$$


Then there exists $\left.\left.\bar{h}_{s, z} \in\right] 0, \min \left\{\frac{1}{4} \eta\left(\frac{\varepsilon}{4}\right), 1-b\right\}\right]$ satisfying

$$
d\left(z+\int_{s}^{s+\bar{h}_{s, z}} f_{s, z}(\tau) d \tau, C\left(s+\bar{h}_{s, z}\right)\right)<\bar{h}_{s, z} \frac{\varepsilon}{4} .
$$

Consider the subset

$$
\begin{aligned}
N(s, z) & =\{(\bar{s}, \bar{z}) \in]-\bar{h}_{s, z}, 1-\bar{h}_{s, z}[\times E: \\
& \left.d\left(\bar{z}+\int_{\bar{s}}^{\bar{s}+\bar{h}_{s, z}} f_{s, z}(\mu) d \mu, C\left(\bar{s}+\bar{h}_{s, z}\right)\right)<\bar{h}_{s, z} \frac{\varepsilon}{4}\right\} .
\end{aligned}
$$

Since $C$ is lower semicontinuous, Corollary 1.2.1 in [1] shows that the function

$$
(\bar{s}, \bar{z}) \mapsto d\left(\bar{z}+\int_{\bar{s}}^{\bar{s}+\bar{h}_{s, z}} f_{s, z}(\mu) d \mu, C\left(\bar{s}+\bar{h}_{s, z}\right)\right)
$$

is upper semicontinuous. So the set $N(s, z)$ is open. Moreover, since $(s, z)$ belongs to $N(s, z)$, there exists $0<\eta_{s, z}<\bar{h}_{s, z}$ such that $] s-\eta_{s, z}, s+\eta_{s, z}\left[\times B\left(z, \eta_{s, z}\right)\right.$ is contained in $N(s, z)$, therefore, the compact subset $M$ can be covered by $q$ such subsets $] s_{i}-\eta_{s_{i}, z_{i}}, s_{i}+\eta_{s_{i}, z_{i}}\left[\times B\left(z_{i}, \eta_{s_{i}, z_{i}}\right)\right.$. For simplicity, we set

$$
h_{i}:=\bar{h}_{s_{i}, z_{i}} \text { and } \eta_{i}:=\eta_{s_{i}, z_{i}}, i=1, \ldots, q \text {. }
$$

Put $\eta=\min \left\{h_{i} / 1 \leq i \leq q\right\}$, take $t \in\left[t_{0}, b_{0}\right]$ and $x \in C(t)$, and let $i \in\{1, \ldots, q\}$ such that $(t, x) \in] s_{i}-\eta_{i}, s_{i}+\eta_{i}\left[\times B\left(z_{i}, \eta_{i}\right)\right.$, hence $(t, x) \in N\left(s_{i}, z_{i}\right)$. So there exists $f_{s_{i}, z_{i}} \in S_{y, \frac{\varepsilon}{4}}\left(z_{i}\right)$ such that

$$
d\left(x+\int_{t}^{t+h_{i}} f_{s_{i}, z_{i}}(\tau) d \tau, C\left(t+h_{i}\right)\right)<h_{i} \frac{\varepsilon}{4} .
$$

On the other hand, let $f_{i} \in S_{f_{s_{i}, z_{i}}, \frac{\varepsilon}{4}}(x)$. By (C2) we have for all $\tau \in\left[t, t+h_{i}\right]$

$\left\|f_{i}(\tau)-f_{s_{i}, z_{i}}(\tau)\right\| \leq d\left(f_{s_{i}, z_{i}}(\tau), F(\tau, x)\right)+\frac{\varepsilon}{4} \leq m(\tau)\left\|x-z_{i}\right\|+\frac{\varepsilon}{4} \leq h_{i} m(\tau)+\frac{\varepsilon}{4}$.

Hence by (2.1) we get

$$
\int_{t}^{t+h_{i}}\left\|f_{i}(\tau)-f_{s_{i}, z_{i}}(\tau)\right\| d \tau \leq h_{i} \int_{t}^{t+h_{i}} m(\tau) d \tau+\frac{h_{i} \varepsilon}{4} \leq \frac{h_{i} \varepsilon}{4}+\frac{h_{i} \varepsilon}{4} \leq \frac{h_{i} \varepsilon}{2} .
$$

Thus

$$
\begin{aligned}
& d\left(x+\int_{t}^{t+h_{i}} f_{i}(\tau) d \tau, C\left(t+h_{i}\right)\right) \\
& \leq d\left(x+\int_{t}^{t+h_{i}} f_{s_{i}, z_{i}}(\tau) d \tau, C\left(t+h_{i}\right)\right)+\int_{t}^{t+h_{i}}\left\|f_{s_{i}, z_{i}}(\tau)-f_{i}(\tau)\right\| d \tau
\end{aligned}
$$




$$
\begin{aligned}
& \leq \frac{h_{i} \varepsilon}{4}+\frac{h_{i} \varepsilon}{2} \\
& \leq \frac{3 h_{i} \varepsilon}{4} .
\end{aligned}
$$

Let $x_{i} \in C\left(t+h_{i}\right)$ such that

$$
\frac{1}{h_{i}}\left\|x_{i}-x-\int_{t}^{t+h_{i}} f_{i}(\tau) d \tau\right\| \leq \frac{1}{h_{i}} d\left(x+\int_{t}^{t+h_{i}} f_{i}(\tau) d \tau, C\left(t+h_{i}\right)\right)+\frac{\varepsilon}{4} \leq \varepsilon .
$$

Set

then $u \in \varepsilon B$ and

$$
u=\frac{1}{h_{i}}\left(x_{i}-x-\int_{t}^{t+h_{i}} f_{i}(\tau) d \tau\right)
$$

$$
x_{i}=\left(x+h_{i} u+\int_{t}^{t+h_{i}} f_{i}(\tau) d \tau\right) \in C\left(t+h_{i}\right) .
$$

Note that $f_{i} \in S_{y,(2 m(.)+1) \varepsilon}(x)$. Indeed, one has

$$
\begin{aligned}
\left\|f_{i}(\tau)-y(\tau)\right\| & \leq\left\|f_{i}(\tau)-f_{s_{i}, z_{i}}(\tau)\right\|+\left\|f_{s_{i}, z_{i}}(\tau)-y(\tau)\right\| \\
& \leq m(\tau) h_{i}+\frac{\varepsilon}{4}+d\left(y(\tau), F\left(\tau, z_{i}\right)\right)+\frac{\varepsilon}{4} \\
& \leq m(\tau) h_{i}+\frac{2 \varepsilon}{4}+d(y(\tau), F(\tau, x))+m(\tau)\left\|x-z_{i}\right\| \\
& \leq m(\tau) \varepsilon+\frac{2 \varepsilon}{4}+d(y(\tau), F(\tau, x))+m(\tau) h_{i} \\
& \leq d(y(\tau), F(\tau, x))+(2 m(\tau)+1) \varepsilon .
\end{aligned}
$$

The proof of the next Lemma is similar to that of Lemma 3.

Lemma 4. If assumptions (L1)-(L3) are satisfied, then for all $0<\varepsilon<a$, for all $t_{0} \in[0, b]$ and for all measurable function $y():.[0, b] \rightarrow E$, there exists $\eta>0(\eta<\varepsilon)$ such that $\forall(t, x) \in\left[t_{0}, b_{0}\right] \times K$, there exist $f \in S_{y,(2 m(.)+1) \varepsilon}(x), h_{t, x} \in\left[\eta, \frac{1}{4} \eta\left(\frac{\varepsilon}{4}\right)\right]$ and $u \in \varepsilon B$ such that

$$
\left(x+h_{t, x} u+\int_{t}^{t+h_{t, x}} f(s) d s\right) \in P(x) .
$$

We need the following Propositions in the next sections.

Proposition 1. If assumptions (C1)-(C3) are satisfied, then for all $0<\varepsilon<a$, $t_{0} \in[0, b], z_{0} \in C\left(t_{0}\right)$ and $y(.) \in L^{1}([0, b], E)$, there exist a continuous mapping $z():.\left[t_{0}, b_{0}\right] \rightarrow E$, a step function $\theta():.\left[t_{0}, b_{0}\right] \rightarrow\left[t_{0}, b_{0}\right]$ and $f(.) \in L^{1}\left(\left[t_{0}, b_{0}\right], E\right)$ such that 
(i) $z\left(t_{0}\right)=z_{0}, \quad f(t) \in F(t, z(\theta(t))), \quad 0 \leq t-\theta(t) \leq \frac{1}{4} \eta\left(\frac{\varepsilon}{4}\right)$ and $z(\theta(t)) \in$ $C(\theta(t))$, for all $t \in\left[t_{0}, b_{0}\right]$

(ii) $\|f(t)-y(t)\| \leq d(y(t), F(t, z(\theta(t))))+(2 m(t)+1) \varepsilon$ for all $t \in\left[t_{0}, b_{0}\right]$;

(iii) $\left\|z(t)-z\left(t_{0}\right)-\int_{t_{0}}^{t} f(\tau) d \tau\right\| \leq \varepsilon\left(t-t_{0}\right)$ for all $t \in\left[t_{0}, b_{0}\right]$.

Proof. Let $0<\varepsilon<a, t_{0} \in[0, b], z_{0} \in C\left(t_{0}\right)$ and $y(.) \in L^{1}([0, b], E)$ be fixed. By Lemma 3, there exist $h_{0} \in\left[\eta, \frac{1}{4} \eta\left(\frac{\varepsilon}{4}\right)\right], f_{0} \in S_{y,(2 m(.)+1) \varepsilon}\left(z_{0}\right)$ and $u_{0} \in \varepsilon B$ such that

$$
\left(z_{0}+h_{0} u_{0}+\int_{t_{0}}^{t_{0}+h_{0}} f_{0}(s) d s\right) \in C\left(t_{0}+h_{0}\right)
$$

Set

$$
z_{1}=z_{0}+h_{0} u_{0}+\int_{t_{0}}^{t_{0}+h_{0}} f_{0}(s) d s \text { and } t_{1}=t_{0}+h_{0}
$$

We reiterate this process for constructing finite sequences $\left(h_{i}\right)_{i} \subset\left[\eta, \frac{1}{4} \eta\left(\frac{\varepsilon}{4}\right)\right]$, $\left(f_{i}(.)\right)_{i},\left(t_{i}\right)_{i},\left(u_{i}\right)_{i} \subset \varepsilon B$, and $\left(z_{i}\right)_{i}$ satisfying the following assertions for $i \geq 0$ :

(a) $t_{i+1}=t_{i}+h_{i}$ and $z_{i+1} \in C\left(t_{i+1}\right)$

(b) $z_{i+1}=z_{i}+h_{i} u_{i}+\int_{t_{i}}^{t_{i}+1} f_{i}(s) d s$;

(c) $f_{i} \in S_{y,(2 m(.)+1) \varepsilon}\left(z_{i}\right)$.

It is easy to see that for $i=0$ the assertions (a)-(c) are fulfilled. Let now $i \geq 1$. Assume that (a)-(c) are satisfied for any $i=1, \ldots, q$. If $b_{0} \leq t_{q+1}$, then we stop the process of iterations and we get that (a)-(c) are satisfied with $t_{q}<b_{0} \leq t_{q+1}$. In the other case, for $\left(t_{q+1}, z_{q+1}\right)$, by Lemma 3, there exist $h_{q+1} \in\left[\eta, \frac{1}{4} \eta\left(\frac{\varepsilon}{4}\right)\right], f_{q+1} \in$ $S_{y,(2 m(.)+1) \varepsilon}\left(z_{q+1}\right)$ and $u_{q+1} \in \varepsilon B$ such that

$$
\left(z_{q+1}+h_{q+1} u_{q+1}+\int_{t_{q+1}}^{t_{q+1}+h_{q+1}} f_{q+1}(s) d s\right) \in C\left(t_{q+1}+h_{q+1}\right) .
$$

Set

$$
z_{q+2}=z_{q+1}+h_{q+1} u_{q+1}+\int_{t_{q+1}}^{t_{q+1}+h_{q+1}} f_{q+1}(s) d s \text { and } t_{q+2}=t_{q+1}+h_{q+1} \text {. }
$$

Thus the conditions (a)-(c) are satisfied for $i=q+1$. Since $h_{i} \geq \eta>0$, there exists an integer $s$ such that $t_{s}<b_{0} \leq t_{s+1}$. Now, we define the function $\theta():.\left[t_{0}, b_{0}\right] \rightarrow\left[t_{0}, b_{0}\right]$ and $f(),. u(.) \in L^{1}\left(\left[t_{0}, b_{0}\right], E\right)$ by setting for all $t \in\left[t_{q}, t_{q+1}\left[\cap\left[t_{0}, b_{0}\right]\right.\right.$

$$
\begin{gathered}
\theta(t)=t_{q}, f(t)=f_{q}(t), u(t)=u_{q}, \\
\theta\left(b_{0}\right)=b_{0}, f\left(b_{0}\right)=f_{s+1}\left(b_{0}\right), u\left(b_{0}\right)=u_{s+1} \text { if } t_{s+1}=b_{0} .
\end{gathered}
$$


Also we define the function $z():.\left[t_{0}, b_{0}\right] \rightarrow E$ by $z\left(t_{0}\right)=z_{0}$ and

$$
z(t)=z\left(t_{0}\right)+\int_{t_{0}}^{t} f(s) d s+\int_{t_{0}}^{t} u(s) d s, \forall t \in\left[t_{0}, b_{0}\right] .
$$

These definitions will enable us to derive assertions (i) and (ii). Moreover, we have for all $t \in\left[t_{0}, b_{0}\right]$

$$
\left\|z(t)-z\left(t_{0}\right)-\int_{t_{0}}^{t} f(s) d s\right\| \leq \varepsilon\left(t-t_{0}\right) .
$$

The proof of Proposition 1 is complete.

The same proof of Proposition 1 holds for the following Proposition.

Proposition 2. If assumptions (L1)-(L3) are satisfied, then for every $\varepsilon \in] 0, a[$, $t_{0} \in[0, b], z_{0} \in K$ and $y(.) \in L^{1}([0, b], E)$, there exist $z(.) \in \mathcal{C}\left(\left[t_{0},+\infty[, E), \theta(),. \tilde{\theta}():.\left[t_{0},+\infty\left[\rightarrow\left[t_{0},+\infty\left[\right.\right.\right.\right.\right.\right.$ and $f(.) \in L^{1}\left(\left[t_{0}, b_{0}\right], E\right)$ such that

(i) $z\left(t_{0}\right)=z_{0}, \quad f(t) \in F(t, z(\theta(t))), 0 \leq t-\theta(t) \leq \frac{1}{4} \eta\left(\frac{\varepsilon}{4}\right), 0 \leq \tilde{\theta}(t)-t \leq$ $\frac{1}{4} \eta\left(\frac{\varepsilon}{4}\right)$ and $z(\tilde{\theta}(t)) \in P(z(\theta(t)))$ for all $t \in\left[t_{0}, b_{0}\right]$;

(ii) $\|f(t)-y(t)\| \leq d(y(t), F(t, z(\theta(t))))+(2 m(t)+1) \varepsilon$ for all $t \in\left[t_{0}, b_{0}\right]$;

(iii) $\left\|z(t)-z\left(t_{0}\right)-\int_{t_{0}}^{t} f(\tau) d \tau\right\| \leq \varepsilon\left(t-t_{0}\right)$ for all $t \in\left[t_{0}, b_{0}\right]$.

Remark that, in this case, $\tilde{\theta}$ and $\theta$ are defined as follows: $\tilde{\theta}(t)=t_{q+1}$ and $\theta(t)=t_{q}$ for all $t \in\left[t_{q}, t_{q+1}[\right.$.

\section{Proof of Theorem 1}

Set $\varphi(0)=z_{0}$ and let $T_{1}>0$ be such that

$$
\int_{0}^{T_{1}} m(t) d t<1
$$

Put $T=\inf \left\{T_{1}, b\right\}$.

We shall show the following Proposition. It will be used in order to obtain a sequence of approximated solutions.

Proposition 3. If assumptions (H1)-(H3) are satisfied, then for all $0<\varepsilon<a$ and for all $y(.) \in L^{1}([0, b], E)$, there exist a continuous mapping $z():.[-a, T] \rightarrow E$, step functions $\theta(),. \bar{\theta}():.[0, T] \rightarrow[0, T], \Gamma():.[0, T] \rightarrow \mathcal{C}_{a}$ and $f(.) \in L^{1}([0, T], E)$ such that

(i) $f(t) \in F(t, \Gamma(t))$ and $z(\theta(t)) \in C(\theta(t))$, for all $t \in[0, T]$ and $z \equiv \varphi$ on $[-a, 0]$

(ii) $0 \leq t-\theta(t) \leq \frac{1}{4} \eta\left(\frac{\varepsilon}{4}\right)$ and $0 \leq t-\bar{\theta}(t) \leq \frac{1}{4} \eta\left(\frac{\varepsilon}{4}\right)$ for all $t \in[0, T]$;

(iii) $\|f(t)-y(t)\| \leq d(y(t), F(t, \Gamma(t)))+(2 m(t)+1) \varepsilon$ for all $t \in[0, T]$; 
(iv) $\left\|z(t)-z(0)-\int_{0}^{t} f(\tau) d \tau\right\| \leq \varepsilon t$ for all $t \in[0, T]$.

(v) For all $t \in[0, T]$

$$
\Gamma(t)(s)= \begin{cases}z\left(\bar{\theta}(t)+\frac{1}{4} \eta\left(\frac{\varepsilon}{4}\right)+s\right) & -a \leq s \leq-\frac{1}{4} \eta\left(\frac{\varepsilon}{4}\right) \\ -\frac{4 s}{\eta\left(\frac{\varepsilon}{4}\right)} z(\bar{\theta}(t))+\left(1+\frac{4 s}{\eta\left(\frac{\varepsilon}{4}\right)}\right) z(\theta(t)) & -\frac{1}{4} \eta\left(\frac{\varepsilon}{4}\right) \leq s \leq 0 .\end{cases}
$$

Proof. Let $0<\varepsilon<a$ and $y(.) \in L^{1}([0, b], E)$ be fixed. Set $t_{0}=0$ and put

$$
z(t)=\varphi(t), \forall t \in[-a, 0] \text {. }
$$

Consider the function $\Gamma_{0}: E \rightarrow \ell_{a}$ defined by

$$
\Gamma_{0}(x)(s)= \begin{cases}z\left(t_{0}+\frac{1}{4} \eta\left(\frac{\varepsilon}{4}\right)+s\right) & -a \leq s \leq-\frac{1}{4} \eta\left(\frac{\varepsilon}{4}\right) \\ -\frac{4 s}{\eta\left(\frac{\varepsilon}{4}\right)} z\left(t_{0}\right)+\left(1+\frac{4 s}{\eta\left(\frac{\varepsilon}{4}\right)}\right) x & -\frac{1}{4} \eta\left(\frac{\varepsilon}{4}\right) \leq s \leq 0,\end{cases}
$$

for all $x \in E$. The set-valued map $G_{0}: G r(C) \rightarrow 2^{E}$ defined by $G_{0}(t, x)=$ $F\left(t, \Gamma_{0}(x)\right)$ satisfies all assumptions (C1)-(C3). Then by Proposition 1, there exist a continuous mapping $z_{0}():.\left[t_{0}, t_{0}+\frac{1}{4} \eta\left(\frac{\varepsilon}{4}\right)\right] \cap[0, T] \rightarrow E$, a step function $\theta_{0}($.$) :$ $\left[t_{0}, t_{0}+\frac{1}{4} \eta\left(\frac{\varepsilon}{4}\right)\right] \cap[0, T] \rightarrow\left[t_{0}, t_{0}+\frac{1}{4} \eta\left(\frac{\varepsilon}{4}\right)\right] \cap[0, T]$ and $f_{0}(.) \in L^{1}\left(\left[t_{0}, t_{0}+\frac{1}{4} \eta\left(\frac{\varepsilon}{4}\right)\right] \cap\right.$ $[0, T], E)$ such that

(i) $z_{0}\left(t_{0}\right)=z_{0}, \quad f_{0}(t) \in F\left(t, \Gamma_{0}\left(z_{0}\left(\theta_{0}(t)\right)\right)\right), \quad 0 \leq t-\theta_{0}(t) \leq \frac{1}{4} \eta\left(\frac{\varepsilon}{4}\right)$ and $z_{0}\left(\theta_{0}(t)\right) \in C\left(\theta_{0}(t)\right)$, for all $t \in\left[t_{0}, t_{0}+\frac{1}{4} \eta\left(\frac{\varepsilon}{4}\right)\right] \cap[0, T]$

(ii) $\left\|f_{0}(t)-y(t)\right\| \leq d\left(y(t), F\left(t, \Gamma_{0}\left(z_{0}\left(\theta_{0}(t)\right)\right)\right)\right)+(2 m(t)+1) \varepsilon$ for all $t \in$ $\left[t_{0}, t_{0}+\frac{1}{4} \eta\left(\frac{\varepsilon}{4}\right)\right] \cap[0, T]$

(iii) $\left\|z_{0}(t)-z_{0}\left(t_{0}\right)-\int_{t_{0}}^{t} f_{0}(\tau) d \tau\right\| \leq \varepsilon\left(t-t_{0}\right)$ for all $t \in\left[t_{0}, t_{0}+\frac{1}{4} \eta\left(\frac{\varepsilon}{4}\right)\right] \cap[0, T]$. Set $t_{1}=t_{0}+\frac{1}{4} \eta\left(\frac{\varepsilon}{4}\right)$ and $z(t)=z_{0}(t)$, for all $t \in\left[t_{0}, t_{1}\right] \cap[0, T]$.

We reiterate this process for constructing sequences $z_{i}():.\left[t_{i}, t_{i+1}\right] \cap[0, T] \rightarrow E$, $\theta_{i}():.\left[t_{i}, t_{i+1}\right] \cap[0, T] \rightarrow\left[t_{i}, t_{i+1}\right] \cap[0, T], \Gamma_{i}: E \rightarrow \mathcal{C}_{a}$ and $f_{i}(.) \in L^{1}\left(\left[t_{i}, t_{i+1}\right] \cap\right.$ $[0, T], E)$ and a continuous function $z():.\left[-a, t_{i+1}\right] \cap[-a, T] \rightarrow E$ satisfying the following assertions for $i \geq 0$ :

(a) $z\left(t_{i}\right)=z_{i}\left(t_{i}\right), \quad f_{i}(t) \in F\left(t, \Gamma_{i}\left(z_{i}\left(\theta_{i}(t)\right)\right)\right), \quad 0 \leq t-\theta_{i}(t) \leq \frac{1}{4} \eta\left(\frac{\varepsilon}{4}\right)$ and $z_{i}\left(\theta_{i}(t)\right) \in C\left(\theta_{i}(t)\right)$, for all $t \in\left[t_{i}, t_{i+1}\right] \cap[0, T]$;

(b) $\left\|f_{i}(t)-y(t)\right\| \leq d\left(y(t), F\left(t, \Gamma_{i}\left(z_{i}\left(\theta_{i}(t)\right)\right)\right)\right)+(2 m(t)+1) \varepsilon$ for all $t \in$ $\left[t_{i}, t_{i+1}\right] \cap[0, T] ;$

(c) $\left\|z_{i}(t)-z_{i}\left(t_{i}\right)-\int_{t_{i}}^{t} f_{i}(\tau) d \tau\right\| \leq \varepsilon\left(t-t_{i}\right)$ for all $t \in\left[t_{i}, t_{i+1}\right] \cap[0, T]$.

(d) For all $x \in E$

$$
\Gamma_{i}(x)(s)= \begin{cases}z\left(t_{i}+\frac{1}{4} \eta\left(\frac{\varepsilon}{4}\right)+s\right) & -a \leq s \leq-\frac{1}{4} \eta\left(\frac{\varepsilon}{4}\right) \\ -\frac{4 s}{\eta\left(\frac{\varepsilon}{4}\right)} z\left(t_{i}\right)+\left(1+\frac{4 s}{\eta\left(\frac{\varepsilon}{4}\right)}\right) x & -\frac{1}{4} \eta\left(\frac{\varepsilon}{4}\right) \leq s \leq 0,\end{cases}
$$


The assertions (a)-(d) are fulfilled for $i=0$. Let now $i \geq 1$. Assume that (a)-(d) are satisfied for any $i=1, \ldots, q$. If $T \leq t_{q+1}$, then we stop this process of iterations and we get that (a)-(d) are satisfied with $t_{q}<T \leq t_{q+1}$. In the other case $t_{q+1}<T$, consider the function $\Gamma_{q+1}: E \rightarrow \mathcal{C}_{a}$ defined by

$$
\Gamma_{q+1}(x)(s)= \begin{cases}z\left(t_{q+1}+\frac{1}{4} \eta\left(\frac{\varepsilon}{4}\right)+s\right) & -a \leq s \leq-\frac{1}{4} \eta\left(\frac{\varepsilon}{4}\right) \\ -\frac{4 s}{\eta\left(\frac{\varepsilon}{4}\right)} z\left(t_{q+1}\right)+\left(1+\frac{4 s}{\eta\left(\frac{\varepsilon}{4}\right)}\right) x & -\frac{1}{4} \eta\left(\frac{\varepsilon}{4}\right) \leq s \leq 0,\end{cases}
$$

for all $x \in E$. The set-valued map $G_{q+1}: G r(C) \rightarrow 2^{E}$ defined by $G_{q+1}(t, x)=$ $F\left(t, \Gamma_{q+1}(x)\right)$ satisfies all assumptions (C1)-(C3). Then by Proposition 1, there exist a continuous mapping $z_{q+1}():.\left[t_{q+1}, t_{q+1}+\frac{1}{4} \eta\left(\frac{\varepsilon}{4}\right)\right] \cap[0, T] \rightarrow E$, a step function $\theta_{q+1}():.\left[t_{q+1}, t_{q+1}+\frac{1}{4} \eta\left(\frac{\varepsilon}{4}\right)\right] \cap[0, T] \rightarrow\left[t_{q+1}, t_{q+1}+\frac{1}{4} \eta\left(\frac{\varepsilon}{4}\right)\right] \cap[0, T]$ and $f_{q+1}(.) \in$ $L^{1}\left(\left[t_{q+1}, t_{q+1}+\frac{1}{4} \eta\left(\frac{\varepsilon}{4}\right)\right] \cap[0, T], E\right)$ satisfying the assertions (a)-(d) for $i=q+1$. Set $t_{q+2}=t_{q+1}+\frac{1}{4} \eta\left(\frac{\varepsilon}{4}\right)$ and $z(t)=z_{q+1}(t)$ for all $t \in\left[t_{q+1}, t_{q+2}\right] \cap[0, T]$. Thus the conditions (a)-(d) are satisfied for $i=q+1$. Since $t_{i+1}-t_{i}=\frac{1}{4} \eta\left(\frac{\varepsilon}{4}\right)$, there exists an integer $s$ such that $t_{s}<T \leq t_{s+1}$. Further on, we define the functions $\theta(),. \bar{\theta}():.[0, T] \rightarrow[0, T], \Gamma:[0, T] \rightarrow \mathcal{C}_{a}$ and $f(.) \in L^{1}([0, T], E)$ by setting for all $t \in\left[t_{q}, t_{q+1}[\cap[0, T]\right.$

$$
\theta(t)=\theta_{q}(t), f(t)=f_{q}(t), \bar{\theta}(t)=t_{q}, \Gamma(t)=\Gamma_{q}\left(z_{q}\left(\theta_{q}(t)\right)\right)
$$

and if $t_{s+1}=T$

$$
\theta(T)=\theta_{s+1}(T), f(T)=f_{s+1}(T), \bar{\theta}(T)=T, \Gamma(T)=\Gamma_{s+1}\left(z_{s+1}\left(\theta_{s+1}(T)\right)\right) .
$$

Moreover, for all $t \in[0, T]$, there exists $0 \leq q \leq s$ such that $t \in\left[t_{q}, t_{q+1}\right] \cap[0, T]$. Then

$$
\begin{aligned}
& \left\|z(t)-z_{0}-\int_{0}^{t} f(s) d s\right\| \\
& \leq\left\|z(t)-z\left(t_{q}\right)-\int_{t_{q}}^{t} f_{q}(s) d s\right\|+\left\|z\left(t_{q}\right)-z_{0}-\int_{t_{0}}^{t_{q}} f(s) d s\right\| \\
& \leq \varepsilon\left(t-t_{q}\right)+\sum_{i=1}^{q}\left\|z\left(t_{i}\right)-z\left(t_{i-1}\right)-\int_{t_{i-1}}^{t_{i}} f_{i-1}(s) d s\right\| \\
& \leq \varepsilon\left(t-t_{q}\right)+\sum_{i=1}^{q} \varepsilon\left(t_{i}-t_{i-1}\right) \\
& \leq \varepsilon t .
\end{aligned}
$$

Hence the proof of Proposition 3 is complete.

Now we are prepared to prove our Theorem 1 . Let $\left(\varepsilon_{n}\right)_{n \geq 1}$ be a strictly decreasing sequence of positive scalars such that $0<\varepsilon_{n}<a$ for all $n \geq 1$ and $\sum_{n=1}^{\infty} \varepsilon_{n}<$ 
$\infty$. In view of Proposition 3, we can define inductively sequences $\left(f_{n}(.)\right)_{n \geq 1} \subset$ $L^{1}([0, T], E), \quad\left(z_{n}(.)\right)_{n \geq 1} \subset \mathcal{C}([-a, T], E), \quad\left(\Gamma_{n}(.)\right)_{n \geq 1} \subset \mathcal{C}\left([0, T], \mathcal{C}_{a}\right)$ and $\left(\theta_{n}(.)\right)_{n \geq 1},\left(\bar{\theta}_{n}(.)\right)_{n \geq 1} \subset S([0, T],[0, T])$ where $S([0, T],[0, T])$ denotes the space of step functions from $[0, T]$ into $[0, T]$ such that

(1) $f_{n}(t) \in F\left(t, \Gamma_{n}(t)\right)$ and $z_{n}\left(\theta_{n}(t)\right) \in C\left(\theta_{n}(t)\right)$, for all $t \in[0, T]$ and $z_{n} \equiv \varphi$ on $[-a, 0]$;

(2) $0 \leq t-\theta_{n}(t) \leq \frac{1}{4} \eta\left(\frac{\varepsilon_{n}}{4}\right)$ and $0 \leq t-\bar{\theta}_{n}(t) \leq \frac{1}{4} \eta\left(\frac{\varepsilon_{n}}{4}\right)$ for all $t \in[0, T]$;

(3) $\left\|f_{n+1}(t)-f_{n}(t)\right\| \leq d\left(f_{n}(t), F\left(t, \Gamma_{n+1}(t)\right)\right)+(2 m(t)+1) \varepsilon_{n+1}$ for all $t \in$ $[0, T]$;

(4) $\left\|z_{n}(t)-z_{0}-\int_{0}^{t} f_{n}(\tau) d \tau\right\| \leq \varepsilon_{n} t$ for all $t \in[0, T]$.

(5) For all $t \in[0, T]$

$$
\Gamma_{n}(t)(s)= \begin{cases}z_{n}\left(\bar{\theta}_{n}(t)+\frac{1}{4} \eta\left(\frac{\varepsilon_{n}}{4}\right)+s\right) & -a \leq s \leq-\frac{1}{4} \eta\left(\frac{\varepsilon_{n}}{4}\right) \\ -\frac{4 s}{\eta\left(\frac{\varepsilon_{n}}{4}\right)} z_{n}\left(\bar{\theta}_{n}(t)\right)+\left(1+\frac{4 s}{\eta\left(\frac{\varepsilon_{n}}{4}\right)}\right) z_{n}\left(\theta_{n}(t)\right) & -\frac{1}{4} \eta\left(\frac{\varepsilon_{n}}{4}\right) \leq s \leq 0 .\end{cases}
$$

From (1) and (3), we deduce for all $t \in[0, T]$

$$
\begin{aligned}
\left\|f_{n+1}(t)-f_{n}(t)\right\| & \leq H\left(F\left(t, \Gamma_{n}(t)\right), F\left(t, \Gamma_{n+1}(t)\right)\right)+(2 m(t)+1) \varepsilon_{n+1} \\
& \leq m(t)\left\|\Gamma_{n}(t)-\Gamma_{n+1}(t)\right\|_{\infty}+(2 m(t)+1) \varepsilon_{n+1} .
\end{aligned}
$$

Claim 1. For all $t \in[0, T]$,

- $\left\|\Gamma_{n}(t)-\Gamma_{n+1}(t)\right\|_{\infty} \leq\left\|z_{n}(.)-z_{n+1}(.)\right\|_{\infty}+12 \varepsilon_{n}$.

- $\left\|T(t) z_{n}-\Gamma_{n}(t)\right\|_{\infty} \leq 8 \varepsilon_{n}$.

Proof. First, remark that for all $t, \bar{t} \in[-a, T]$ and $\rho>0$ such that $|t-\bar{t}| \leq \eta(\rho)$ we have $\left\|z_{n}(t)-z_{n}(\bar{t})\right\| \leq 2\left(\varepsilon_{n}+\rho\right)$. Indeed, let $t, \bar{t} \in[-a, T]$ such that $|t-\bar{t}| \leq \eta(\rho)$. If $t, \bar{t} \in[0, T]$ and $\bar{t} \leq t$, one has

$$
\begin{aligned}
& \left\|z_{n}(t)-z_{n}(\bar{t})\right\| \\
& \leq\left\|z_{n}(t)-z_{0}-\int_{0}^{t} f_{n}(s) d s\right\|+\left\|z_{n}(\bar{t})-z_{0}-\int_{0}^{\bar{t}} f_{n}(s) d s\right\| \\
& +\int_{\bar{t}}^{t}\left\|f_{n}(s)\right\| d s \\
& \leq \varepsilon_{n} t+\varepsilon_{n} \bar{t}+\int_{\bar{t}}^{t} g(s) d s \\
& \leq 2 \varepsilon_{n}+\rho .
\end{aligned}
$$

If $t, \bar{t} \in[-a, 0]$, by construction, we have

$$
\left\|z_{n}(t)-z_{n}(\bar{t})\right\|=\|\varphi(t)-\varphi(\bar{t})\| \leq \rho .
$$


If $t \in[0, T]$ and $\bar{t} \in[-a, 0]$ one has $|t| \leq \eta(\rho)$ and $|\bar{t}| \leq \eta(\rho)$. Then

$$
\begin{aligned}
& \left\|z_{n}(t)-z_{n}(\bar{t})\right\| \\
& \leq\left\|z_{n}(t)-z_{0}-\int_{0}^{t} f_{n}(s) d s\right\|+\|\varphi(\bar{t})-\varphi(0)\|+\int_{0}^{t}\left\|f_{n}(s)\right\| d s \\
& \leq \varepsilon_{n} t+\rho+\rho \\
& \leq \varepsilon_{n}+2 \rho .
\end{aligned}
$$

Hence we conclude that for all $t, \bar{t} \in[-a, T]$ such that $|t-\bar{t}| \leq \eta(\rho)$, we have $\left\|z_{n}(t)-z_{n}(\bar{t})\right\| \leq 2\left(\varepsilon_{n}+\rho\right)$.

Now, let $t \in[0, T]$ and $-a \leq s \leq-\frac{1}{4} \eta\left(\frac{\varepsilon_{n}}{4}\right)$. We have

$$
\begin{aligned}
& \left|\bar{\theta}_{n+1}(t)+\frac{1}{4} \eta\left(\frac{\varepsilon_{n+1}}{4}\right)+s-\bar{\theta}_{n}(t)-\frac{1}{4} \eta\left(\frac{\varepsilon_{n}}{4}\right)-s\right| \\
& \leq\left|\bar{\theta}_{n+1}(t)-t\right|+\left|\bar{\theta}_{n}(t)-t\right|+\frac{1}{4} \eta\left(\frac{\varepsilon_{n+1}}{4}\right)+\frac{1}{4} \eta\left(\frac{\varepsilon_{n}}{4}\right) \\
& \leq \frac{1}{4} \eta\left(\frac{\varepsilon_{n+1}}{4}\right)+\frac{1}{4} \eta\left(\frac{\varepsilon_{n}}{4}\right)+\frac{1}{4} \eta\left(\frac{\varepsilon_{n}}{4}\right)+\frac{1}{4} \eta\left(\frac{\varepsilon_{n}}{4}\right) \\
& \leq \eta\left(\frac{\varepsilon_{n}}{4}\right) .
\end{aligned}
$$

Thus

$$
\begin{aligned}
& \left\|\Gamma_{n+1}(t)(s)-\Gamma_{n}(t)(s)\right\| \\
& =\left\|z_{n+1}\left(\bar{\theta}_{n+1}(t)+\frac{1}{4} \eta\left(\frac{\varepsilon_{n+1}}{4}\right)+s\right)-z_{n}\left(\bar{\theta}_{n}(t)+\frac{1}{4} \eta\left(\frac{\varepsilon_{n}}{4}\right)+s\right)\right\| \\
& \leq\left\|z_{n+1}\left(\bar{\theta}_{n+1}(t)+\frac{1}{4} \eta\left(\frac{\varepsilon_{n+1}}{4}\right)+s\right)-z_{n+1}\left(\bar{\theta}_{n}(t)+\frac{1}{4} \eta\left(\frac{\varepsilon_{n}}{4}\right)+s\right)\right\| \\
& +\left\|z_{n+1}\left(\bar{\theta}_{n}(t)+\frac{1}{4} \eta\left(\frac{\varepsilon_{n}}{4}\right)+s\right)-z_{n}\left(\bar{\theta}_{n}(t)+\frac{1}{4} \eta\left(\frac{\varepsilon_{n}}{4}\right)+s\right)\right\| \\
& \leq 2\left(\varepsilon_{n+1}+\frac{\varepsilon_{n}}{4}\right)+\left\|z_{n+1}(.)-z_{n}(.)\right\|_{\infty} \\
& \leq 4 \varepsilon_{n}+\left\|z_{n+1}(.)-z_{n}(.)\right\|_{\infty} .
\end{aligned}
$$

If $-\frac{1}{4} \eta\left(\frac{\varepsilon_{n}}{4}\right) \leq s \leq-\frac{1}{4} \eta\left(\frac{\varepsilon_{n+1}}{4}\right)$ we get

$$
\begin{aligned}
\left|\bar{\theta}_{n+1}(t)+\frac{1}{4} \eta\left(\frac{\varepsilon_{n+1}}{4}\right)+s-\theta_{n}(t)\right| & \leq\left|\bar{\theta}_{n+1}(t)-t\right|+\left|\theta_{n}(t)-t\right|+\frac{1}{4} \eta\left(\frac{\varepsilon_{n+1}}{4}\right)+|s| \\
& \leq \frac{1}{4} \eta\left(\frac{\varepsilon_{n+1}}{4}\right)+\frac{1}{4} \eta\left(\frac{\varepsilon_{n}}{4}\right)+\frac{1}{4} \eta\left(\frac{\varepsilon_{n}}{4}\right)+\frac{1}{4} \eta\left(\frac{\varepsilon_{n}}{4}\right) \\
& \leq \eta\left(\frac{\varepsilon_{n}}{4}\right) .
\end{aligned}
$$


Then

$$
\begin{aligned}
& \left\|\Gamma_{n+1}(t)(s)-\Gamma_{n}(t)(s)\right\| \\
& =\left\|z_{n+1}\left(\bar{\theta}_{n+1}(t)+\frac{1}{4} \eta\left(\frac{\varepsilon_{n+1}}{4}\right)+s\right)+\frac{4 s}{\eta\left(\frac{\varepsilon_{n}}{4}\right)} z_{n}\left(\bar{\theta}_{n}(t)\right)-\left(1+\frac{4 s}{\eta\left(\frac{\varepsilon_{n}}{4}\right)}\right) z_{n}\left(\theta_{n}(t)\right)\right\| \\
& \leq\left\|z_{n+1}\left(\bar{\theta}_{n+1}(t)+\frac{1}{4} \eta\left(\frac{\varepsilon_{n+1}}{4}\right)+s\right)-z_{n}\left(\theta_{n}(t)\right)\right\|+\left\|z_{n}\left(\bar{\theta}_{n}(t)\right)-z_{n}\left(\theta_{n}(t)\right)\right\| \\
& \leq\left\|z_{n+1}\left(\bar{\theta}_{n+1}(t)+\frac{1}{4} \eta\left(\frac{\varepsilon_{n+1}}{4}\right)+s\right)-z_{n}\left(\bar{\theta}_{n+1}(t)+\frac{1}{4} \eta\left(\frac{\varepsilon_{n+1}}{4}\right)+s\right)\right\| \\
& +\left\|z_{n}\left(\bar{\theta}_{n+1}(t)+\frac{1}{4} \eta\left(\frac{\varepsilon_{n+1}}{4}\right)+s\right)-z_{n}\left(\theta_{n}(t)\right)\right\|+\left\|z_{n}\left(\bar{\theta}_{n}(t)\right)-z_{n}\left(\theta_{n}(t)\right)\right\| \\
& \leq\left\|z_{n+1}(.)-z_{n}(.)\right\|_{\infty}+2\left(\varepsilon_{n}+\frac{\varepsilon_{n}}{4}\right)+2\left(\varepsilon_{n}+\frac{\varepsilon_{n}}{4}\right) \\
& \leq\left\|z_{n+1}(.)-z_{n}(.)\right\|_{\infty}+8 \varepsilon_{n} . \\
\text { If } & -\frac{1}{4} \eta\left(\frac{\varepsilon_{n+1}}{4}\right) \leq s \leq 0 \text { one has } \\
& \left\|\Gamma_{n+1}(t)(s)-\Gamma_{n}(t)(s)\right\| \\
& \leq \frac{4|s|}{\eta\left(\frac{\varepsilon_{n+1}}{4}\right)}\left\|z_{n+1}\left(\bar{\theta}_{n+1}(t)\right)-z_{n+1}\left(\theta_{n+1}(t)\right)\right\|+\frac{4|s|}{\eta\left(\frac{\varepsilon_{n}}{4}\right)}\left\|z_{n}\left(\bar{\theta}_{n}(t)\right)-z_{n}\left(\theta_{n}(t)\right)\right\| \\
& +\left\|z_{n+1}\left(\theta_{n+1}(t)\right)-z_{n}\left(\theta_{n}(t)\right)\right\| \\
& \leq\left\|z_{n+1}\left(\bar{\theta}_{n+1}(t)\right)-z_{n+1}\left(\theta_{n+1}(t)\right)\right\|+\left\|z_{n}\left(\bar{\theta}_{n}(t)\right)-z_{n}\left(\theta_{n}(t)\right)\right\| \\
& +\left\|z_{n+1}\left(\theta_{n+1}(t)\right)-z_{n}\left(\theta_{n}(t)\right)\right\| \\
& \leq 2\left(\varepsilon_{n+1}+\frac{\varepsilon_{n}}{4}\right)+2\left(\varepsilon_{n}+\frac{\varepsilon_{n}}{4}\right) \\
& +\left\|z_{n+1}\left(\theta_{n+1}(t)\right)-z_{n+1}\left(\theta_{n}(t)\right)\right\|+\left\|z_{n+1}\left(\theta_{n}(t)\right)-z_{n}\left(\theta_{n}(t)\right)\right\| \\
& \leq 8 \varepsilon_{n}+2\left(\varepsilon_{n+1}+\frac{\varepsilon_{n}}{4}\right)+\left\|z_{n+1}(.)-z_{n}(.)\right\| \infty \\
& \leq\left\|z_{n+1}(.)-z_{n}(.)\right\|_{\infty}+12 \varepsilon_{n} . \\
&
\end{aligned}
$$

Thus we conclude that $\left\|\Gamma_{n+1}(t)-\Gamma_{n}(t)\right\|_{\infty} \leq\left\|z_{n+1}(.)-z_{n}(.)\right\|_{\infty}+12 \varepsilon_{n}$.

By the same arguments as above, for $t \in[0, T]$, if $-a \leq s \leq-\frac{1}{4} \eta\left(\frac{\varepsilon_{n}}{4}\right)$ we have

$$
\begin{aligned}
\left|\bar{\theta}_{n}(t)+\frac{1}{4} \eta\left(\frac{\varepsilon_{n}}{4}\right)+s-t-s\right| & \leq\left|\bar{\theta}_{n}(t)-t\right|+\frac{1}{4} \eta\left(\frac{\varepsilon_{n}}{4}\right) \\
& \leq \frac{1}{4} \eta\left(\frac{\varepsilon_{n}}{4}\right)+\frac{1}{4} \eta\left(\frac{\varepsilon_{n}}{4}\right) \\
& \leq \eta\left(\frac{\varepsilon_{n}}{4}\right) .
\end{aligned}
$$

Then

$$
\left\|T(t) z_{n}(s)-\Gamma_{n}(t)(s)\right\|
$$




$$
\begin{aligned}
& =\left\|z_{n}(t+s)-z_{n}\left(\bar{\theta}_{n}(t)+\frac{1}{4} \eta\left(\frac{\varepsilon_{n}}{4}\right)+s\right)\right\| \\
& \leq 2\left(\varepsilon_{n}+\frac{\varepsilon_{n}}{4}\right) \\
& \leq 4 \varepsilon_{n} .
\end{aligned}
$$

If $-\frac{1}{4} \eta\left(\frac{\varepsilon_{n}}{4}\right) \leq s \leq 0$ we get

$$
\begin{aligned}
\left|\theta_{n}(t)-t-s\right| & \leq\left|\theta_{n}(t)-t\right|+|s| \\
& \leq \frac{1}{4} \eta\left(\frac{\varepsilon_{n}}{4}\right)+\frac{1}{4} \eta\left(\frac{\varepsilon_{n}}{4}\right) \\
& \leq \eta\left(\frac{\varepsilon_{n}}{4}\right)
\end{aligned}
$$

So

$$
\begin{aligned}
& \left\|T(t) z_{n}(s)-\Gamma_{n}(t)(s)\right\| \\
& =\left\|z_{n}(t+s)+\frac{4 s}{\eta\left(\frac{\varepsilon_{n}}{4}\right)} z_{n}\left(\bar{\theta}_{n}(t)\right)-\left(1+\frac{4 s}{\eta\left(\frac{\varepsilon_{n}}{4}\right)}\right) z_{n}\left(\theta_{n}(t)\right)\right\| \\
& \leq\left\|z_{n}(t+s)-z_{n}\left(\theta_{n}(t)\right)\right\|+\left\|z_{n}\left(\bar{\theta}_{n}(t)\right)-z_{n}\left(\theta_{n}(t)\right)\right\| \\
& \leq 2\left(\varepsilon_{n}+\frac{\varepsilon_{n}}{4}\right)+2\left(\varepsilon_{n}+\frac{\varepsilon_{n}}{4}\right) \\
& \leq 8 \varepsilon_{n} .
\end{aligned}
$$

Thus we conclude that $\left\|T(t) z_{n}-\Gamma_{n}(t)\right\|_{\infty} \leq 8 \varepsilon_{n}$.

Now, Relations (4.1), (4.2) and Claim 1 yield for all $t \in[0, T]$

$$
\begin{aligned}
& \left\|z_{n+1}(t)-z_{n}(t)\right\| \\
& \leq\left\|z_{n+1}(t)-z_{0}-\int_{0}^{t} f_{n+1}(s) d s\right\|+\int_{0}^{t}\left\|f_{n+1}(s)-f_{n}(s)\right\| d s \\
& +\left\|z_{n}(t)-z_{0}-\int_{0}^{t} f_{n}(s) d s\right\| \\
& \leq \varepsilon_{n+1} t+\varepsilon_{n} t+\left\|z_{n}(.)-z_{n+1}(.)\right\|_{\infty} \int_{0}^{t} m(s) d s+12 \varepsilon_{n} \int_{0}^{t} m(s) d s \\
& +2 \varepsilon_{n+1} \int_{0}^{t} m(s) d s+t \varepsilon_{n+1} \\
& \leq 2 \varepsilon_{n} t+\left\|z_{n}(.)-z_{n+1}(.)\right\|_{\infty} \int_{0}^{T} m(s) d s+12 \varepsilon_{n}+2 \varepsilon_{n}+t \varepsilon_{n} \\
& \leq 17 \varepsilon_{n}+\left\|z_{n}(.)-z_{n+1}(.)\right\|_{\infty} \int_{0}^{T} m(s) d s .
\end{aligned}
$$


It follows that

$$
\left\|z_{n}(.)-z_{n+1}(.)\right\|_{\infty} \leq \frac{17 \varepsilon_{n}}{1-L},
$$

where $L=\int_{0}^{T} m(s) d s$. Therefore we have, for $n<m$

$$
\left\|z_{m}(.)-z_{n}(.)\right\|_{\infty} \leq \frac{17}{1-L} \sum_{i=n}^{m-1} \varepsilon_{i}
$$

Thus the sequence $\left(z_{n}(.)\right)_{n \geq 1}$ converges uniformly on $[-a, T]$ to a function $x($.$) .$ Also, since all functions $z_{n}($.$) agree with \varphi($.$) on [-a, 0]$, we have $x(.) \equiv \varphi($.$) on$ $[-a, 0]$.

Additionally, observe that $z_{n}\left(\theta_{n}(t)\right)$ converges uniformly to $x(t)$ on $[0, T]$. Indeed, for $t \in[0, T]$, we have $\left|t-\theta_{n}(t)\right| \leq \frac{1}{4} \eta\left(\frac{\varepsilon_{n}}{4}\right)$ then

$$
\begin{aligned}
\left\|z_{n}(t)-z_{n}\left(\theta_{n}(t)\right)\right\| & \leq 2\left(\varepsilon_{n}+\frac{\varepsilon_{n}}{4}\right) \\
& \leq 4 \varepsilon_{n} .
\end{aligned}
$$

Hence $\left\|z_{n}(t)-z_{n}\left(\theta_{n}(t)\right)\right\|$ converges to 0 as $n \rightarrow \infty$. Since

$$
\left\|z_{n}\left(\theta_{n}(t)\right)-x(t)\right\| \leq\left\|z_{n}(t)-z_{n}\left(\theta_{n}(t)\right)\right\|+\left\|z_{n}(t)-x(t)\right\|
$$

and $\left(z_{n}().\right)$ converges uniformly to $x($.$) , it follows z_{n}\left(\theta_{n}(t)\right)$ converges uniformly to $x(t)$ on $[0, T]$. By construction, we have $z_{n}\left(\theta_{n}(t)\right) \in C\left(\theta_{n}(t)\right)$ for every $t \in[0, T]$ and the graph of $C$ is closed, then $x(t) \in C(t)$ for all $t \in[0, T]$. Furthermore, the uniform convergence of $z_{n}($.$) to x($.$) on [-a, T]$ implies that $T(t) z_{n}$ converges to $T(t) x$ uniformly on $[-a, 0]$. From Claim 1 we deduce that

$$
\Gamma_{n}(t) \text { converges to } T(t) x \text { in } e_{a} \text {. }
$$

Now, we return to Relation (4.2). By Claim 1 and Relation (4.3) we have for all $t \in[0, T]$

$$
\begin{aligned}
\left\|f_{n+1}(t)-f_{n}(t)\right\| & \leq m(t)\left\|\Gamma_{n}(t)-\Gamma_{n+1}(t)\right\|_{\infty}+(2 m(t)+1) \varepsilon_{n+1} \\
& \leq m(t)\left(\left\|z_{n}(.)-z_{n+1}(.)\right\|_{\infty}+12 \varepsilon_{n}\right)+(2 m(t)+1) \varepsilon_{n} \\
& \leq m(t)\left(\frac{17 \varepsilon_{n}}{1-L}+12 \varepsilon_{n}\right)+(2 m(t)+1) \varepsilon_{n} \\
& \leq\left(m(t)\left(\frac{17}{1-L}+14\right)+1\right) \varepsilon_{n} .
\end{aligned}
$$

This implies (as above) that $\left(f_{n}(t)\right)_{n \geq 1}$ is a Cauchy sequence and $\left(f_{n}(.)\right)_{n \geq 1}$ converges point-wisely to $f($.$) . Further, since \left\|f_{n}(t)\right\| \leq g(t)$, by (4) and by the Lebesgue dominated convergence theorem, we have

$$
x(t)=\lim _{n \rightarrow \infty} z_{n}(t)=\lim _{n \rightarrow \infty}\left(z_{0}+\int_{0}^{t} f_{n}(s) d s\right)=z_{0}+\int_{0}^{t} f(s) d s .
$$


Finally, observe that by (1),

$$
\begin{aligned}
d(f(t), F(t, T(t) x)) & \leq\left\|f(t)-f_{n}(t)\right\|+H\left(F\left(t, \Gamma_{n}(t)\right), F(t, T(t) x)\right) \\
& \leq\left\|f(t)-f_{n}(t)\right\|+m(t)\left\|\Gamma_{n}(t)-T(t) x\right\|_{\infty} .
\end{aligned}
$$

Since $f_{n}(t)$ converges to $f(t)$ and by (4.4) the last term converges to 0 . So that $\dot{x}(t)=f(t) \in F(t, T(t) x)$ a.e in $[0, T]$. The proof is complete.

\section{Proof of TheOREM 2}

Set $\varphi(0)=z_{0}$. We emphasize that the choice of $T$ and $\eta(\varepsilon)$ are as in the last sections.

The same proof of Proposition 3 holds for the following Proposition. We shall apply it in order to construct a sequence of approximated solutions.

Proposition 4. If assumptions (L1)-(L3) are satisfied, then for all $0<\varepsilon<a$ and $y(.) \in L^{1}([0, b], E)$, there exist a continuous mapping $z():.[-a,+\infty[\rightarrow E$, step functions $\theta(),. \tilde{\theta}(),. \bar{\theta}():.\left[0,+\infty\left[\rightarrow\left[0,+\infty\left[, \Gamma():.[0, T] \rightarrow \mathcal{C}_{a}\right.\right.\right.\right.$, and $f(.) \in$ $L^{1}([0, T], E)$ such that

(i) $f(t) \in F(t, \Gamma(t))$ and $z(\tilde{\theta}(t)) \in P(z(\theta(t)))$, for all $t \in[0, T]$ and $z \equiv \varphi$ on $[-a, 0]$

(ii) $0 \leq t-\theta(t) \leq \frac{1}{4} \eta\left(\frac{\varepsilon}{4}\right), 0 \leq \tilde{\theta}(t)-t \leq \frac{1}{4} \eta\left(\frac{\varepsilon}{4}\right)$ and $0 \leq t-\bar{\theta}(t) \leq \frac{1}{4} \eta\left(\frac{\varepsilon}{4}\right)$ for all $t \in[0, T]$

(iii) $\|f(t)-y(t)\| \leq d(y(t), F(t, \Gamma(t)))+(2 m(t)+1) \varepsilon$ for all $t \in[0, T]$;

(iv) $\left\|z(t)-z(0)-\int_{0}^{t} f(\tau) d \tau\right\| \leq \varepsilon t$ for all $t \in[0, T]$.

(v) For all $t \in[0, T]$

$$
\Gamma(t)(s)= \begin{cases}z\left(\bar{\theta}(t)+\frac{1}{4} \eta\left(\frac{\varepsilon}{4}\right)+s\right) & -a \leq s \leq-\frac{1}{4} \eta\left(\frac{\varepsilon}{4}\right) \\ -\frac{4 s}{\eta\left(\frac{\varepsilon}{4}\right)} z(\theta(t))+\left(1+\frac{4 s}{\eta\left(\frac{\varepsilon}{4}\right)}\right) z(\theta(t)) & -\frac{1}{4} \eta\left(\frac{\varepsilon}{4}\right) \leq s \leq 0 .\end{cases}
$$

Now, let $\left(\varepsilon_{n}\right)_{n \geq 1}$ be a strictly decreasing sequence of positive scalars such that $0<\varepsilon_{n}<a$ for all $n \geq 1$ and $\sum_{n=1}^{\infty} \varepsilon_{n}<\infty$. In view of Proposition 4, we can define inductively sequences $\left(f_{n}(.)\right)_{n \geq 1} \subset L^{1}([0, T], E),\left(z_{n}(.)\right)_{n \geq 1} \subset \mathcal{C}([-a,+\infty[, E)$, $\left(\Gamma_{n}(.)\right)_{n \geq 1} \subset \mathcal{C}\left([0, T], \mathcal{C}_{a}\right)$ and $\left(\theta_{n}(.)\right)_{n \geq 1},\left(\tilde{\theta}_{n}(.)\right)_{n \geq 1},\left(\bar{\theta}_{n}(.)\right)_{n \geq 1} \subset S([0,+\infty[$, $[0,+\infty[)$ such that

(1) $f_{n}(t) \in F\left(t, \Gamma_{n}(t)\right)$ and $z_{n}\left(\tilde{\theta}_{n}(t)\right) \in P\left(z\left(\theta_{n}(t)\right)\right)$, for all $t \in[0, T]$ and $z_{n} \equiv \varphi$ on $[-a, 0]$

(2) $0 \leq t-\theta_{n}(t) \leq \frac{1}{4} \eta\left(\frac{\varepsilon_{n}}{4}\right), 0 \leq \tilde{\theta}_{n}(t)-t \leq \frac{1}{4} \eta\left(\frac{\varepsilon_{n}}{4}\right)$ and $0 \leq t-\bar{\theta}_{n}(t) \leq \frac{1}{4} \eta\left(\frac{\varepsilon_{n}}{4}\right)$ for all $t \in[0, T]$

(3) $\left\|f_{n+1}(t)-f_{n}(t)\right\| \leq d\left(f_{n}(t), F\left(t, \Gamma_{n+1}(t)\right)\right)+(2 m(t)+1) \varepsilon_{n+1}$ for all $t \in$ $[0, T]$ 
(4) $\left\|z_{n}(t)-z_{0}-\int_{0}^{t} f_{n}(\tau) d \tau\right\| \leq \varepsilon_{n} t$ for all $t \in[0, T]$.

(5) For all $t \in[0, T]$

$$
\Gamma_{n}(t)(s)= \begin{cases}z_{n}\left(\bar{\theta}_{n}(t)+\frac{1}{4} \eta\left(\frac{\varepsilon_{n}}{4}\right)+s\right) & -a \leq s \leq-\frac{1}{4} \eta\left(\frac{\varepsilon_{n}}{4}\right) \\ -\frac{4 s}{\eta\left(\frac{\varepsilon_{n}}{4}\right)} z_{n}\left(\bar{\theta}_{n}(t)\right)+\left(1+\frac{4 s}{\eta\left(\frac{\varepsilon_{n}}{4}\right)}\right) z_{n}\left(\theta_{n}(t)\right) & -\frac{1}{4} \eta\left(\frac{\varepsilon_{n}}{4}\right) \leq s \leq 0 .\end{cases}
$$

We can apply the same technique as above to prove that $\left(z_{n}(.)\right)_{n \geq 1}$ converges uniformly to an absolutely continuous function $x($.) and we can deduce that, for almost all $t \in[0, T], \dot{x}(t) \in F(t, T(t) x)$. To complete the proof of Theorem 2, it remains to prove that $x(t) \in P(x(t))$, for all $t \in[0, T]$ and if $t^{\prime}<t$ then $x(t) \in P\left(x\left(t^{\prime}\right)\right)$. Since $z_{n}\left(\tilde{\theta}_{n}(t)\right) \in P\left(z_{n}\left(\theta_{n}(t)\right)\right) \subset K$ for all $t \in[0, T]$ and $K$ is compact, we conclude that $x(t) \in K$ for all $t \in[0, T]$. Then by (A1), $x(t) \in P(x(t))$ for all $t \in[0, T]$. Next, let $t^{\prime}, t \in[0, T]$ such that $t^{\prime}<t$. Then for $n$ large enough we can find $p, q \in\{0, \ldots, s\}$ such that $q=p+i$ where $1 \leq i \leq s, t^{\prime} \in\left[t_{p}^{n}, t_{p+1}^{n}\left[\right.\right.$ and $t \in\left[t_{q}^{n}, t_{q+1}^{n}\left[\right.\right.$, where $t_{i}^{n}$ is defined in the proof of Proposition 4. By construction, we have $z_{n}\left(t_{q-1}^{n}\right) \in P\left(z_{n}\left(t_{q-2}^{n}\right)\right)$, which together with (A1) gives

$$
P\left(z_{n}\left(t_{q-1}^{n}\right)\right) \subseteq P\left(z_{n}\left(t_{q-2}^{n}\right)\right) .
$$

Similarly,

$$
P\left(z_{n}\left(t_{q-2}^{n}\right)\right) \subseteq P\left(z_{n}\left(t_{q-3}^{n}\right)\right) .
$$

If we continue for $i-1$ steps, we obtain

$$
P\left(z_{n}\left(t_{q-1}^{n}\right)\right) \subseteq P\left(z_{n}\left(t_{p}^{n}\right)\right) .
$$

By the fact that $z_{n}\left(t_{q}^{n}\right) \in P\left(z_{n}\left(t_{q-1}^{n}\right)\right)$, we have $z_{n}\left(t_{q}^{n}\right) \in P\left(z_{n}\left(t_{p}^{n}\right)\right)$. This means that $z_{n}\left(\theta_{n}(t)\right) \in P\left(z_{n}\left(\theta_{n}\left(t^{\prime}\right)\right)\right)$. By letting $n \rightarrow+\infty$, we get $x(t) \in P\left(x\left(t^{\prime}\right)\right)$. The proof is complete.

\section{ACKNOWLEDGEMENTS}

The author would like to thank the referee for his careful and thorough reading of the paper.

\section{REFERENCES}

[1] J.-P. Aubin and A. Cellina, Differential inclusions, ser. Grundlehren Math. Wiss. Berlin: Springer-Verlag, 1984, vol. 264.

[2] C. Castaing and M. Valadier, Convex analysis and measurable multifunctions, ser. Lecture Notes in Mathematics. Berlin-New York: Springer-Verlag, 1977, vol. 580.

[3] T. X. Duc Ha, "Existence of viable solutions for nonconvex-valued differential inclusions in Banach spaces," Portugal. Math., vol. 52, no. 2, pp. 241-250, 1995.

[4] A. Gavioli and L. Malaguti, "Viable solutions of differential inclusions with memory in Banach spaces," Portugal. Math., vol. 57, no. 2, pp. 203-217, 2000.

[5] G. Haddad, "Monotone trajectories of differential inclusions and functional-differential inclusions with memory," Israel J. Math., vol. 39, no. 1-2, pp. 83-100, 1981. 
[6] G. Haddad, "Monotone viable trajectories for functional-differential inclusions," J. Differential Equations, vol. 42, no. 1, pp. 1-24, 1981.

[7] M. Larrieu, Invariance d'un fermé pour un champ de vecteurs de Carathéodory. Publications Mathématiques de Pau, 1981.

[8] V. Lupulescu and M. Necula, "A viable result for nonconvex differential inclusions with memory," Port. Math. (N.S.), vol. 63, no. 3, pp. 335-350, 2006.

[9] A. Syam, Contributions aux inclusions différentielles, ser. Doctoral thesis. Université Montpellier II, 1993.

[10] Q. J. Zhu, "On the solution set of differential inclusions in Banach space," J. Differential Equations, vol. 93, no. 2, pp. 213-237, 1991.

\section{Author's address}

\section{Myelkebir Aitalioubrahim}

High school Ibn Khaldoune, BP 13100, commune Bouznika, Morocco.

E-mail address: aitalifrehotmail.com 